\title{
OPTIMAL SENSOR PLACEMENT STRATEGY FOR THE IDENTIFICATION OF LOCAL BOLTED CONNECTION FAILURES IN STEEL STRUCTURES
}

\author{
S. Biswal, Y. Wang* \\ Department of Civil and Environmental Engineering, University of Surrey, United Kingdom \\ * Corresponding author
}

\begin{abstract}
Failure of bolted connections in steel structures may result in catastrophic effects. Many algorithms in existing literature use modal information of a structure to identify damage in that structure, based on the data acquired from accelerometers which record the vibration time histories at different points on the structure. The location of these points may have significant effects on the quality of the acquired data, and thus the identified modal information. In this paper, a distance measure based Markov chain Monte Carlo algorithm is proposed to determine the optimal locations for the accelerometers, and the optimal location of the impact hammer if need. Different damage cases with various combinations of bolt failures are considered in this study. Failures at various levels are simulated by loosening the bolts in a predefined order. To compare the efficiency of the proposed method, the total effect of various damage cases on the accelerations at the optimal locations are calculated for the proposed method and a state-of-the-art method from the existing literature. The results demonstrate the efficiency of the proposed strategy in locating the accelerometers, which can produce data that are more sensitive to the bolted connection failures.
\end{abstract}

\section{Introduction}

Bolted connections with high tensile clamping forces are widely used in steel structures. However, after repetitive external forces and vibrations during the long service life of the structures, the bolted connections experience loss of preloads, i.e., becoming loosened. This may result in the failure of the entire structure. Identification of the loss in pretension is thus essential to ensure the safety of bolted connections and prevent catastrophic failures (Milanese et al. 2008).

The present project considers a steel portal frame (Figure 1) with pre-tensioned bolted connections between the beam and columns. The details about the member sections and the position and numbering of bolts where damage may occur are shown in Figure 2. It aims to use the measured acceleration time signals to identify the loss of pretension at any of the bolt locations. Damage identification algorithms in the time domain will be developed to compare the time signal between the healthy structure and the damaged structure to detect, locate and quantify the damage.

The aim of this paper is to estimate an optimal location of accelerometers where the damage is more sensitive to the measured accelerations. Sensitivity indices can be estimated using distance-based measures reported by Abhinav and Manohar (2016). For a given damage scenario, if the distance between the time signals obtained from the healthy structure and the damaged structure is larger at location 1 than at location 2, then it can be said that the response at location 1 is more sensitive to damage than response at location 2. An optimal sensor placement technique based on modal identification error is presented in Liu et al. (2015). Various types of optimization algorithms are used in Papadimitriou (2005), Guo et al. (2015), and Yuen and Kuok (2015) for estimating optimal sensor location by minimizing information entropy. A hybrid optimization technique using mean square error along with an adaptive genitive algorithm is reported in Chen et al. (2017) for determining the optimal number of sensors and their locations. An optimal sensor placement using a bond energy algorithm is presented in $\mathrm{Lu}$ et al. (2016). A multi-objective algorithm is used in Lin et al. (2018) for estimating multi-type sensor placement on a structure. A sensor placement strategy based on the Fisher information matrix is reported in Zhou et al. (2013), and Meo and Zumpano (2005). A review of methodologies for optimal sensor placement for health monitoring of structures are mentioned in Yi and Li (2012).

\section{Figure 1 Experimental test set-up}

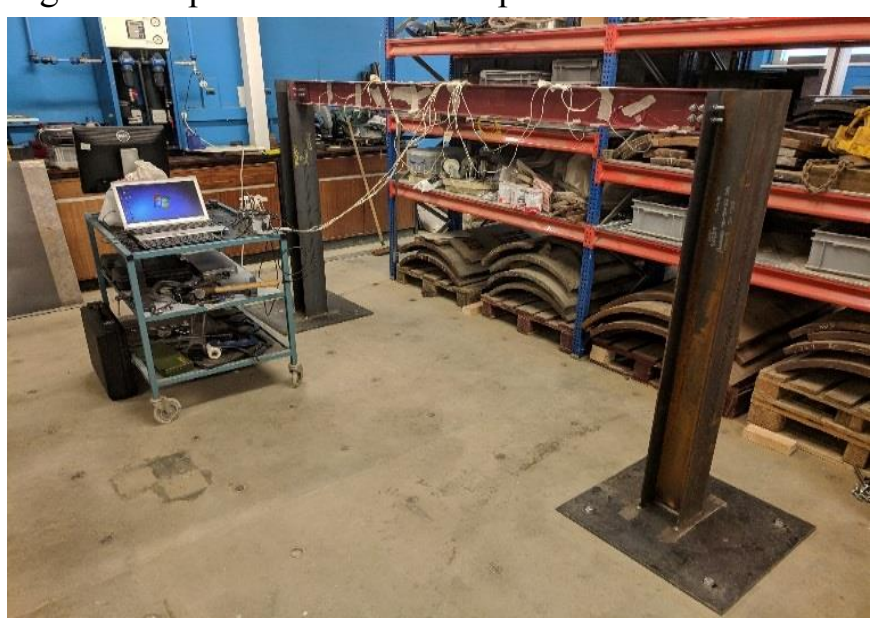


Figure 2 Details of steel members and numbering of bolts

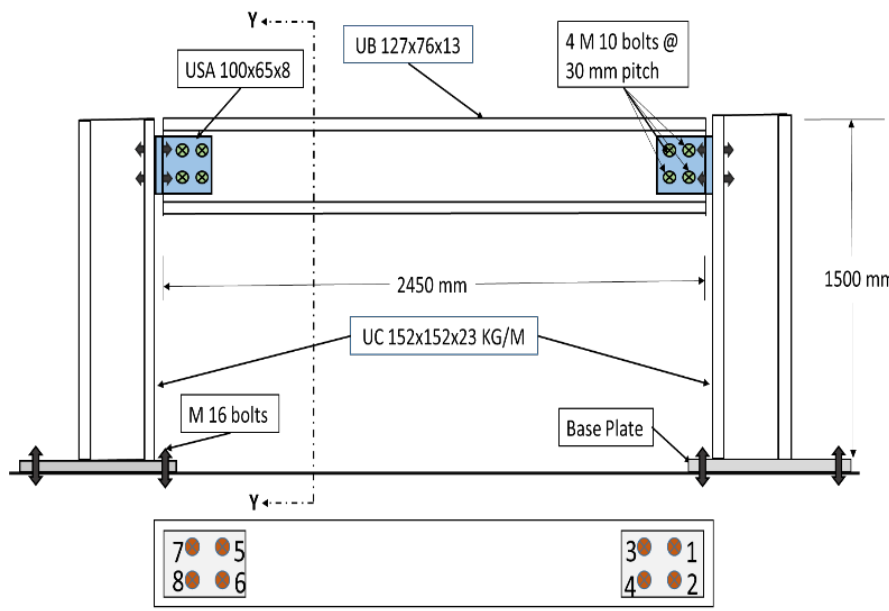

In the present study, a Markov chain Monte Carlo based modified Metropolis-Hastings algorithm reported in Zuev and Katafygiotis (2011) is used to find the optimal location of accelerometers on the steel portal frame. The objective function in the modified Metropolis-Hastings algorithm is the minimization of cumulative LB Keogh distance between time signals obtained from the healthy structure and those obtained from the damaged structure. The LB_Keogh distance used in this study was developed by Keogh and Ratanamahatana (2005). To verify the efficiency of the proposed algorithm, it is then compared with an optimal sensor placement strategy reported in Zhou et al. (2013).

\section{Methodology}

\subsection{LB_Keogh distance metric}

Let us consider two signals in the time domain, signal 1 as ' $\mathrm{S}$ ' and signal 2 as ' $R$ ' as shown in Figure 3. Let the time signals be accelerations measured from a modal test using an impulse hammer. Let signal ' $S$ ' denote acceleration measured from the healthy structure, and signal ' $R$ ' denote acceleration measured from the damaged structure.

Figure 3 Two signals with a shift in time

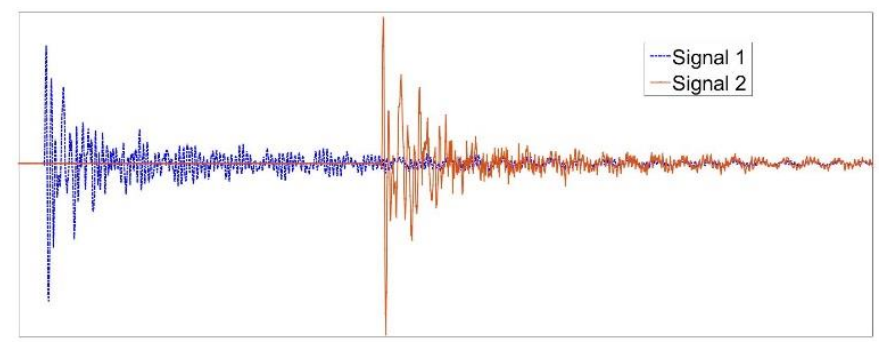

Any anomaly in terms of occurrence of damage can be predicted by comparing the two signals. The Euclidean distance measure is a well-known technique for comparing two signals for any anomaly. In many ways, the Euclidean distance measure present several drawbacks like (i) it is only applicable to time series of equal length, (ii) it does not take into account outliers or noise, (iii) it is very sensitive to signal transformation like shifting, scaling in time or amplitude, time warping etc. Dynamic time warping (DTW) is a distance measure that allows non-linear alignments between two signals, thus it can be applied to time signals of different lengths and at the same time, it is less sensitive to signal transformations. The time shift between two signals (Figure 3 ) is efficiently handled by DTW as shown in Figure 4.

Figure 4 Dynamic time warping for comparison of signals

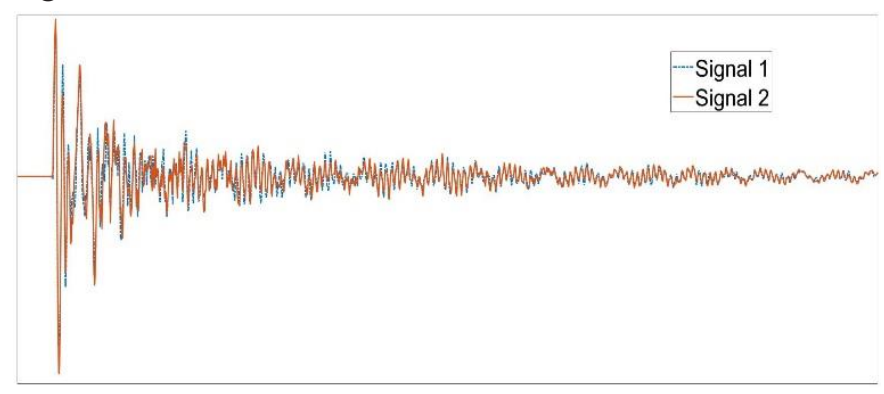

Though the superiority of DTW has long been known, most research until lately implement Euclidean distance-based measure, as it can more efficiently be calculated. As shown in Keogh and Ratanamahatana (2005), a lower bounding function can speed up the calculation from $O\left(n^{2}\right)$ to $O(n)$. It is also shown that DTW with lower bounding can be much faster than Euclidean-based measure. Let us define two sequences UB and LB for a given query sequence ' $S$ ' such that $\forall i, L B_{i} \leq S_{i} \leq U B_{i}$. In practice, uncertainties are embedded in measured time signals. Lower bound $(L B)$ and upper bound $(U B)$ can be taken as the $95 \%$ confidence bounds of the measured time signal ' $S$ '. Let the candidate sequence be ' $R$ '. Lower bounding defined in Keogh and Ratanamahatana (2005) as the square root of the squared sum of the distances from every part of the candidate sequence ' $\mathrm{R}$ ' not falling within the boundary envelope of ' $S$ ', to the nearest orthogonal edge of the boundary envelope. The LB_Koegh distance measure by Keogh and Ratanamahatana (2005), between $\mathrm{S}$ and R (Figure 5) is given by

$\mathrm{LB} \_\operatorname{Keogh}(\mathrm{S}, \mathrm{R})=\sqrt{\sum_{i=1}^{n} \begin{cases}\left(c_{i}-U B_{i}\right)^{2}, & \text { if } c_{i}>U B_{i} \\ \left(c_{i}-L B_{i}\right)^{2}, & \text { if } c_{i}<L B_{i}\end{cases} }$

Figure 5 LB_Keogh distance measure

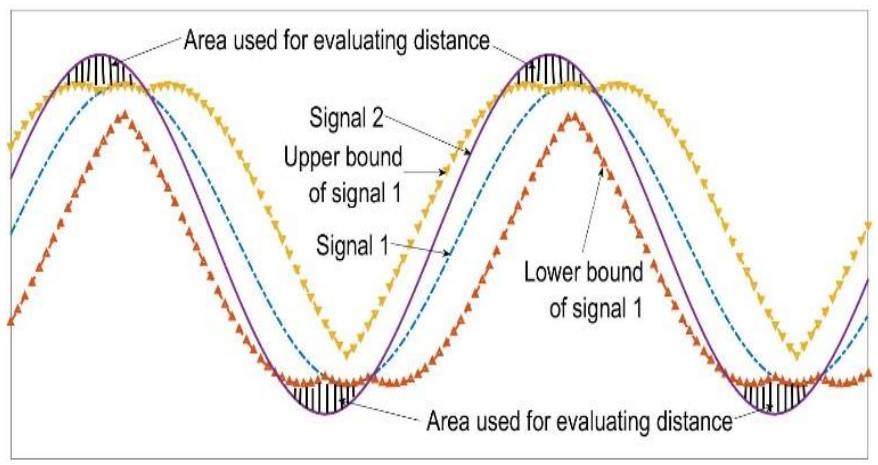




\subsection{Optimal sensor placement with known damage}

Let the structure (here portal frame) be divided into $N$-1 number of elements, such that there are $N$ number of nodes. Let $m$ be the number of accelerometers available for the test. The objective of the study is to find the optimal location of accelerometers on the structure. The accelerometers at the optimal locations maximize the LB Keogh distance between the query sequence measured from the healthy structure and the candidate signal measured from the damaged structure. However, uncertainties are always present in the measurements due to gauge sensitivity, calibration error, quality of data, errors in recording, transmitting and manipulating recorded data. Uncertainties in the measured time signals are propagated to the estimated output data. Hence it is necessary to consider the uncertainties in the measured time signals while estimating the optimal location of the sensors. Let $\mathrm{X}_{m \times 1}$ be a random vector of size $m \times 1$ with probability density function $\pi$, the element of which represents the node number at which an accelerometer is fixed on the structure. $\mathrm{X}$ is defined as $\mathrm{X}_{i} \sim \mathrm{U}_{\text {int }}\left[1, \mathrm{~N}_{n}\right]$, where $i=$ $1 \ldots \mathrm{m}$.

Let $Z_{u}$ be the vector of accelerations measured on the undamaged structure at ' $\mathrm{X}$ ' locations, and $\mathrm{Z}_{d}$ be the vector of accelerations measured on the damaged structure at ' $\mathrm{X}$ ' locations for a given damage scenario. Modified MetropolisHastings algorithm by Zuev and Katafygiotis (2011) is used in this study to simulate samples from the target density $\pi\left(X \mid O_{f}\right)$, where $O_{f}$ is the objective function. In this study, the objective function $O_{f}$ is defined as the maximization of the cumulative LB_Keogh distance (CLB_Keogh) for every measured acceleration data. Using $\mathrm{MMH}$ algorithm separate Markov chains are run for every element of X, and a Markov chain is moved from $j-1^{\text {th }}$ position to $j^{\text {th }}$ position if CLB_Keogh calculated at $j^{\text {th }}$ simulation step is larger than CLB_Keogh at $j-1^{\text {th }}$ simulation step. Markov chains are run till convergence is achieved or the assigned number of samples are covered. For the completeness, the steps of $\mathrm{MMH}$ algorithm are explained below.

[1] Initialize $\mathrm{X}$ as $X_{i}^{0}=U_{\text {int }}\left[1, N_{n}\right], i=1 \ldots m$.

[2] For $j=1 \ldots N_{\text {samp }}$, where $N_{\text {samp }}$ is the number of samples considered in the MMH algorithm

○ For $i=1 \ldots m$

- Simulate $\hat{X}_{i}$ from the proposal density $\pi_{j}\left(\bullet \mid X_{i}^{j-1}\right)$

- Accept $\hat{X}_{i}$ with probability $\alpha_{i}\left(\hat{X}_{i}, X_{i}^{j-1}\right)$, and set $X_{i}=\hat{X}_{i}$

- Or reject $\hat{X}_{i}$ with probability $1-\alpha_{i}\left(\hat{X}_{i}, X_{i}^{j-1}\right)$, and set $X_{i}=X_{i}^{j-1}$
- Return $i$

○ Find LB_Koegh distance measure for each acceleration time signal $X_{i}, X_{i}^{j-1}, i=1 \ldots m$ between the damaged structure and the healthy structure, and then add them to get the cumulative LB_Keogh distance measure CLB_Keogh $(X)$ and CLB_Keogh $\left(X^{j-1}\right)$. LB_Keogh distance measures are also calculated across the measurement points (that represents crosscorrelation between different measured signals) at a given step of the Markov chain, and then added to the CLB_Keogh obtained at the corresponding step of Markov chain.

○ Accept $X$ by setting $X^{j}=X$ if CLB_Keogh $(X)>$ CLB_Keogh $\left(X^{j-1}\right)$.

○ Or reject $X$ by setting $X^{j}=X^{j-1}$

- $\operatorname{Return} j$

The acceptance probability $\alpha_{i}\left(\hat{X}_{i}, X_{i}^{j-1}\right)$ is given by

$\alpha_{i}\left(\hat{X}_{i}, X_{i}^{j-1}\right)=\min \left\{1, \frac{p\left(\hat{X}_{i}\right) \pi_{i}\left(X_{i}^{j-1} \mid \hat{X}_{i}\right)}{p\left(X_{i}^{j-1}\right) \pi_{i}\left(\hat{X}_{i} \mid X_{i}^{j-1}\right)}\right\}$

where $p(\bullet \bullet)$ is the prior probability.

\subsection{Optimal sensor placement with uncertain damage}

For condition assessment and damage identification of structures on site, the prior location of damage and extent of damage are not known. To estimate the optimal location of sensors, in this case, CLB_Keogh is calculated for every damage case. A Markov chain is moved from $j-1^{\text {th }}$ position to $j^{\text {th }}$ position if for every damage case $k\left(k=1 \ldots . \mathrm{N}_{d}\right)$, CLB_Keogh calculated for damage case $k$ at $j^{\text {th }}$ simulation step is larger than CLB_Keogh at $j-1^{\text {th }}$ simulation step for the same damage case $k$. If the number of damage cases $\mathrm{N}_{d}$ is large, it may result in a large number of samples in the Markov chain simulation getting rejected. To avoid a large number of rejections, a Markov chain is allowed to move to the next position if for $\mathrm{N}_{d r}$ number of damage cases (where, $\mathrm{N}_{d r}<\mathrm{N}_{d}$ ) CLB_Keogh $j^{\text {th }}$ simulation step is larger than CLB_Keogh at $j-1^{\text {th }}$ simulation step. $\mathrm{N}_{d r}$ can be chosen (Mourad et al., 2012) such that $P\left(\mathrm{~N}_{d r} \leq \mathrm{n}_{d r} \mid \mathrm{N}_{d}\right)>1-q$ for any $q$ lying between 0 and 1 . The probability $P\left(\mathrm{~N}_{d r} \leq \mathrm{n}_{d r} \mid \mathrm{N}_{d}\right)$ can be derived using discrete binomial distribution as $P\left(\mathrm{~N}_{d r} \leq \mathrm{n}_{d r} \mid \mathrm{N}_{d}\right)=\sum_{r=0}^{\mathrm{n}_{d r}}\left(\begin{array}{l}\mathrm{N}_{d} \\ r\end{array}\right) p^{r}(1-p)^{\mathrm{N}_{d}-r}$, 
where $p$ is the probability of CLB_Keogh calculated for a damage case $k$ at $j^{\text {th }}$ simulation step being larger than CLB_Keogh at $j-1^{\text {th }}$ simulation step for the same damage case.

\subsection{Optimal position of impulse hammer}

For identifying modal parameters of a structure using an impulse hammer and accelerometers, the impulse needs to be applied at each location where accelerometers are fixed. An impulse hammer is usually used to excite the structure. When modal frequencies are only of concern, the impulse should be applied at a position, such that a maximum number of consecutive modes get excited. In this study, the position of the hammer is chosen so that it will maximize CLB_Keogh for accelerations measured at a given set of locations. The position of the hammer is also taken as a random variable (Uniform random integer varying between 1 to the total number of nodes taken for the given structure). A separate Markov chain is added to the MMH algorithm mentioned in the previous section, keeping the objective function the same as before.

\section{Numerical simulation}

\subsection{Finite element model of a steel portal frame}

Opensees by Mazzoni et al. (2009) and Mckenna F et al. (2009), is used in this study for finite element modelling of the steel frame. A total of 50 nodes are assigned to the finite element model as shown in Figure 6, 14 each on the columns and 22 on the beam excluding the two nodes shared with the two columns. The number of nodes in beam and columns is chosen such that the minimum distance between two nodes (that represent minimum distance between two accelerometers) is greater than $100 \mathrm{~mm}$.

Figure 6 Node numbers in the finite element model for beam and columns

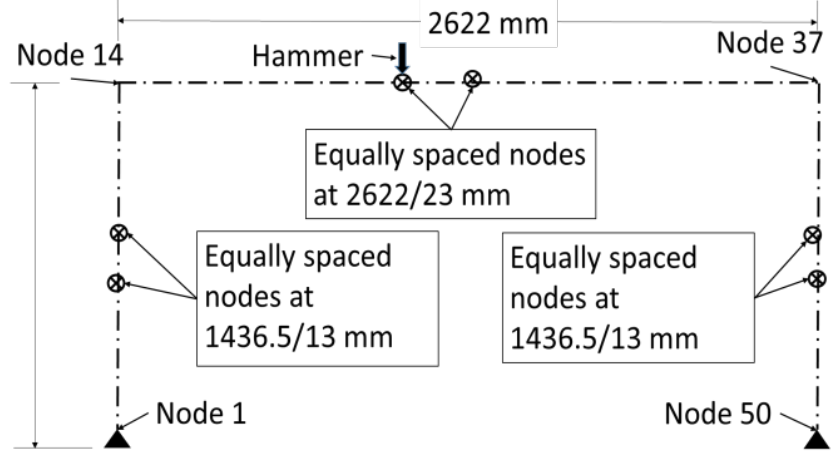

Beam and columns are modelled using 'elasticBeamColumn' elements. Bolts with numbers one to eight as shown in Figure 7 are modelled using a set of springs consisting of (i) one 'zeroLength' element along the length of beam, (ii) one 'zeroLength' element along the depth of beam, and (iii) one 'zeroLength' element along the rotation about the transverse axis.
Figure 7 Bolt positions in the beam

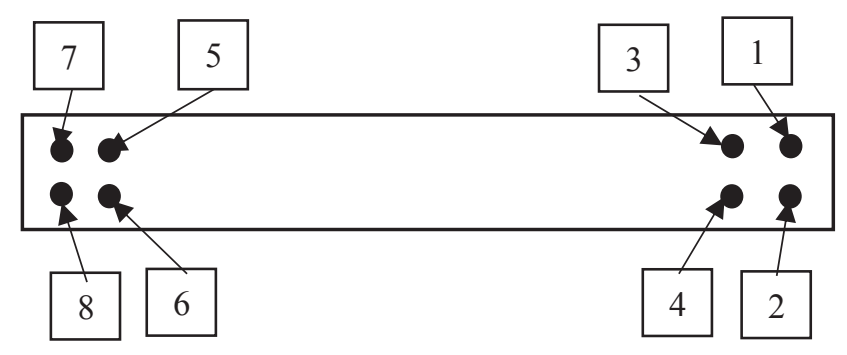

Figure 8 Line sketch of finite element model in Opensees

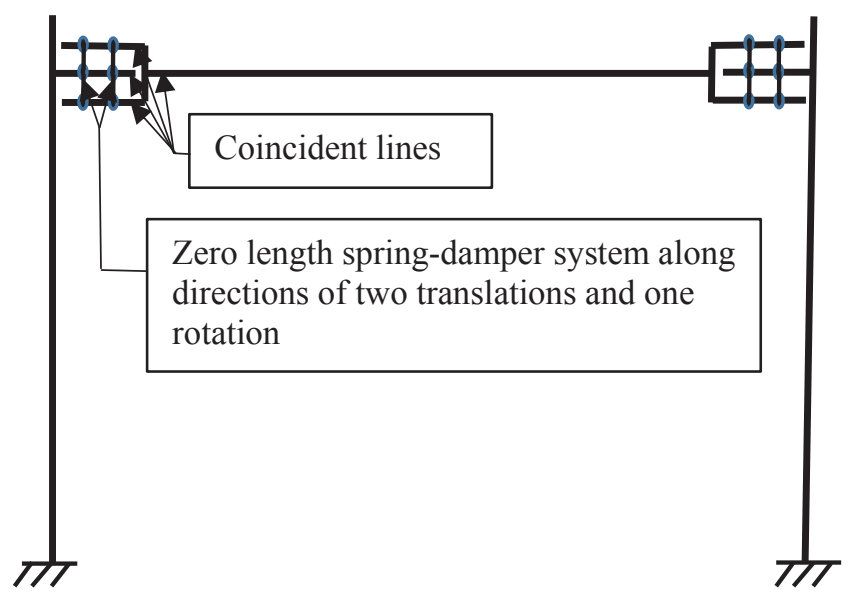

As shown in Figure 8, three coincident lines are used for representing the gusset angle at the centre of beam and beam at two levels of bolts. The bolts in the beam are then connected to the gusset angle using zero length spring-damper elements in Opensees. Loosening of the bolt at a particular position will result in a change in stiffness and damping coefficient of the spring-damper element. The actual change in stiffness and damping coefficient can be identified using finite element model updating, and a future study is undergoing focussing that aspect. The 'zeroLength' elements are assigned with 'Elastic' and 'Viscous' properties to represent three spring mass damper systems for providing restraints along the length, depth and rotation degrees of freedom. The stiffness and viscous properties of the spring damper system are taken as random variables. In the present study a quantitative loosening of a bolt is simulated by changing the spring stiffness by $90 \%$, and at the same time keeping the damping constant as constant. It is understood that the loosening of the bolt will affect both stiffness and damping coefficient of the spring-damper system. However, for introducing small damage only the spring stiffness has been changed in the present study.

\section{Results}

\subsection{Estimated optimal sensor location with known damage}

In this study four damage cases are considered (i) case 1: bolt 1 is fully loosened, (ii) case 2: bolts 1,2 , and 3 are fully loosened, (iii) case 3: bolts 1, 2, 5 and 6 are fully loosened, and (iv) case 4: bolts $1,3,5$ and 7 are fully loosened. The optimal location of accelerometers and position of impulse hammer for 
damage case 1 to damage case 4 are shown in Figure 9 to Figure 12. Here the damage case was known a-priori and the only unknowns were the position of hammer and location of accelerometers.

Figure 9 Optimal location of accelerometers and hammer when bolt 1 is loosened

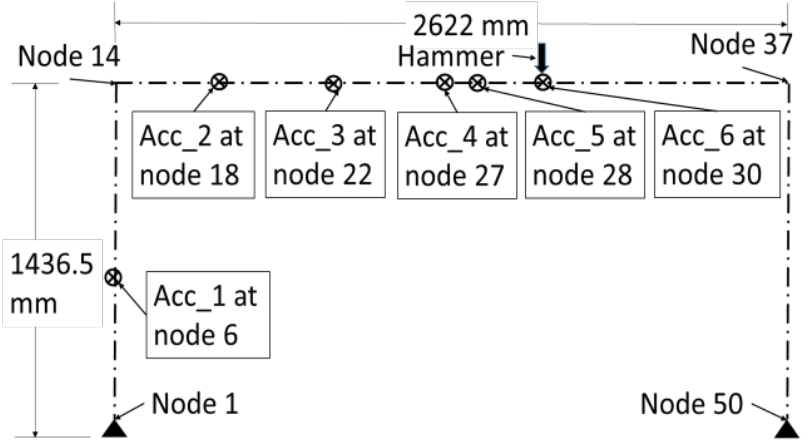

Figure 10 Optimal location of accelerometers and hammer when bolts 1,2 and 3 are loosened

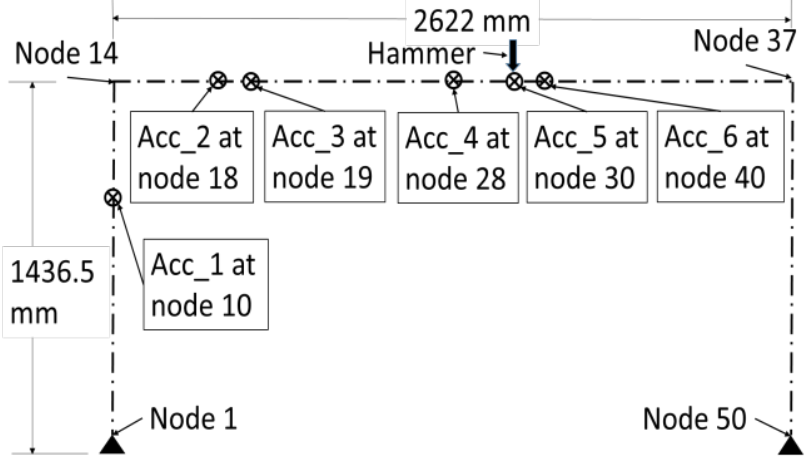

Figure 11 Optimal location of accelerometers and hammer when bolts 1, 3, 5 and 7 are loosened

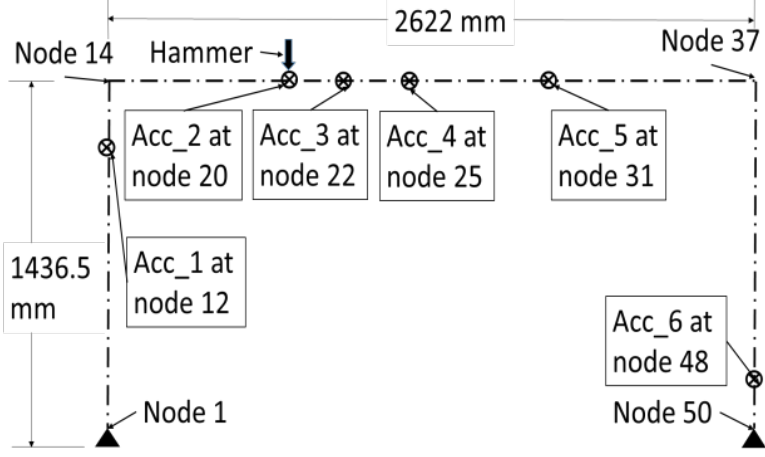

\subsection{Estimated optimal sensor location with unknown damage}

When the damage is not known a-priori, the estimated optimal location of impulse hammer and accelerometers are shown in Figure 13. A summary of the location of the impulse hammer and accelerometers for various damage cases are given in Table 1.
Figure 12 Optimal location of accelerometers and hammer when bolts 1, 2, 5 and 6 are loosened

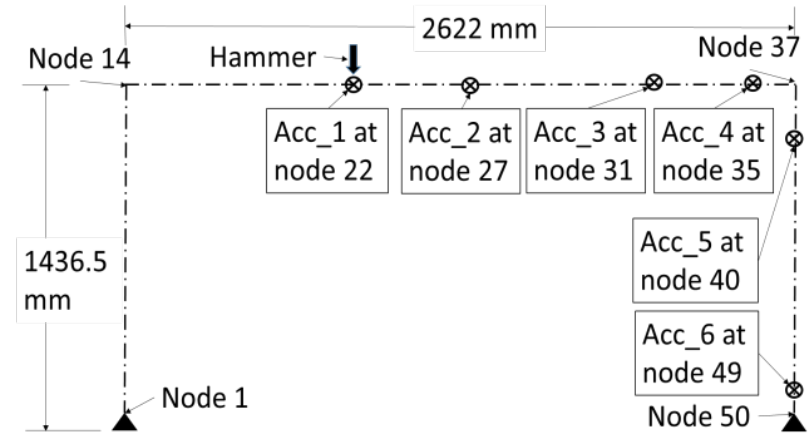

Figure 13 Optimal location of accelerometers and hammer with uncertain locations of loosened bolts

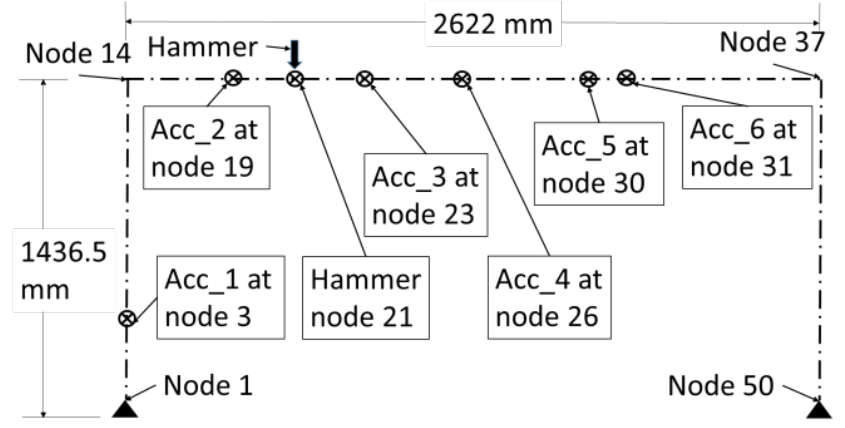

Table 1 Optimal location of accelerometers and hammer by the proposed method

\begin{tabular}{|c|c|c|}
\hline Damage case & Accelerometer locations & $\begin{array}{l}\text { Hammer } \\
\text { location }\end{array}$ \\
\hline Bolt 1 loosened & $6,18,22,27,28,30$ & 30 \\
\hline $\begin{array}{l}\text { Bolts } 1,2,5 \& 6 \\
\text { loosened }\end{array}$ & $22,27,31,35,40,49$ & 22 \\
\hline $\begin{array}{l}\text { Bolts } 1,3,5 \& 7 \\
\text { loosened }\end{array}$ & $12,20,22,25,31,48$ & 20 \\
\hline $\begin{array}{l}\text { Bolts 1, } 2 \& \& 3 \\
\text { loosened }\end{array}$ & $10,18,19,28,30,31$ & 30 \\
\hline $\begin{array}{l}\text { Random location } \\
\text { of bolt loosened }\end{array}$ & $3,19,23,26,30,31$ & 21 \\
\hline
\end{tabular}

\subsection{Comparison with a state-of-the-art method}

The estimated optimal sensor locations are then compared with the optimal sensor locations obtained by Zhou et al. (2013). The optimal location of accelerometers obtained using Zhou et al. (2013) are shown in Figure 14. To compare the efficiency of the proposed optimal location, the total effect of damage on the acceleration time signals are calculated by L2-norm based distance measure as given in Abhinav and Manohar (2016). Greater the value of total effect, higher will be the sensitivity of damage to the output acceleration time signals. The comparison of the estimated total effect for different damage cases given the sensor locations by the proposed method and by Zhou et al. (2013) is given in Table 2. From Table 2, it is clear that for damage case 3 , when bolts $1,3,5$ and 7 are 
loosened, the total effect of the damage on the accelerations measured at the locations obtained by proposed algorithm, and the locations obtained by Zhou et al. (2013) have identical values. However, in all other three damage cases, the total effect for the proposed method is higher than the total effect for the optimal locations obtained using Zhou et al. (2013). The distances measures estimated by the proposed method are at an average of $30 \%$ larger than the distances estimated by Zhou et al. (2013). That means the accelerations measured at the proposed optimal locations are more sensitive to damage.

Figure 14 Accelerometer locations based on damage measurability by Zhou et al. (2013)

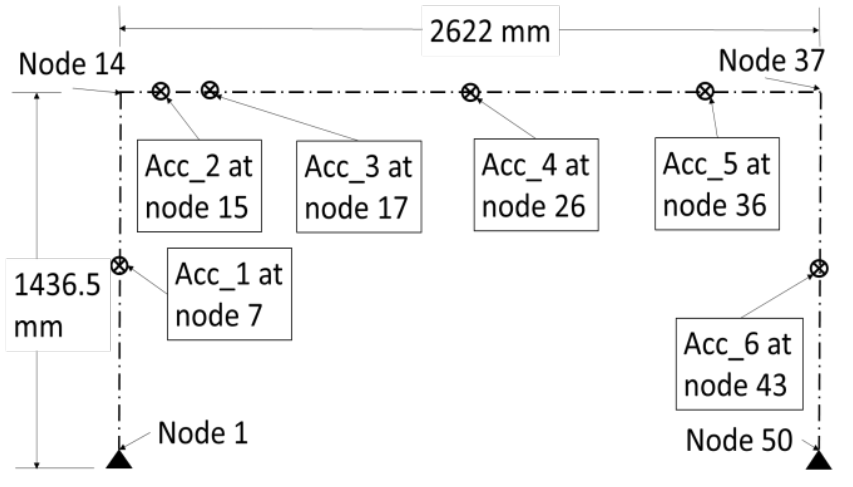

Table 2 Comparison of the proposed method to damage measurability by Zhou et al (2013)

\begin{tabular}{|c|c|c|}
\hline Damage case & $\begin{array}{l}\text { Total effect of } \\
\text { damage case to } \\
\text { acceleration } \\
\text { signals by the } \\
\text { proposed method }\end{array}$ & $\begin{array}{l}\text { Total effect of } \\
\text { damage case to } \\
\text { acceleration } \\
\text { signals by Zhou } \\
\text { et al }(2013)\end{array}$ \\
\hline Bolt 1 loosened & 2429 & 1977 \\
\hline $\begin{array}{l}\text { Bolts } 1,2,5 \& 6 \\
\text { loosened }\end{array}$ & 2349 & 1730 \\
\hline $\begin{array}{l}\text { Bolts } 1,3,5 \& 7 \\
\text { loosened }\end{array}$ & 2580 & 2580 \\
\hline $\begin{array}{l}\text { Bolts } 1,2 \quad \& \quad 3 \\
\text { loosened }\end{array}$ & 3268 & 2622 \\
\hline
\end{tabular}

\section{Conclusion}

This study used a modified Metropolis-Hasting algorithm to determine the optimal location of accelerometers as well as the impact hammer on a portal frame. Optimal sensor locations are estimated for four damage cases when the damage locations and severities are known, and for another case when the location of damage is not known a-priori. To compare the efficiency of the proposed algorithm with that of a state-of-theart method, the total effect of the damage on the accelerations at the optimal locations are calculated. Based on the results, it is clear that the acceleration time signals at the proposed optimal locations are more sensitive to damage than the existing method.

\section{Acknowledgements}

The authors would like to acknowledge Engineering and Physical Sciences Research Council, UK Research and Innovation, for the financial support with Grant Reference Number: EP/R021090/1.

\section{References}

Abhinav S and Manohar CS (2016) Global response sensitivity analysis of randomly excited dynamic structures. Journal of Engineering Mechanics, ASCE 142(3): (04015094) 1-10. https://doi.org/10.1061/(ASCE)EM.1943-7889.0001019

Chen et al. (2017) A hybrid method of optimal sensor placement for dynamic response monitoring of hydrostructures. International Journal of Distributed Sensor Networks, SAGE 13(5): 1-12. DOI: 10.1177/1550147717707728

Guo et al. (2015) Optimal sensor placement for damage detection of bridges subject to ship collision. Structural Control and Health Monitoring, Wiley: 1-16. https://doi.org/10.1002/stc.1963

Keogh E and Ratanamahatana CA (2005) Exact indexing of dynamic time warping. Knowledge and Information Systems, Springer 7(3): 358-386. https://doi.org/10.1007/s10115-0040154-9

Lin et al. (2018) Structural damage detection-oriented multitype sensor placement with multi-objective optimization. Journal of Sound and Vibration, Elsevier 422: 568-589. https://doi.org/10.1016/j.jsv.2018.01.047

Liu et al. (2015) Optimum wireless sensor deployment scheme for structural health monitoring: A simulation study. Smart Materials and Structures, IOP Publishing 24(11): 1-12. DOI: 10.1088/0964-1726/24/11/115034

Lu et al. (2016) Data correlation analysis for optimal sensor placement using a bond energy algorithm. Measurement, Elsevier 91: 509-518. https://doi.org/10.1016/j.measurement.2016.05.089

Mckenna F et al. (2009) Nonlinear Finite-Element Analysis Software Architecture Using Object Composition. Journal of Computing in Civil Engineering, ASCE 24(1): 95-107, https://doi.org/10.1061/(ASCE)CP.1943-5487.0000002

Mazzoni S et al. (2009) Open System for Earthquake Engineering Simulation, User Command-Language Manual. Pacific Earthquake Engineering Research Center, University of California, Berkeley, OpenSees version 2.0.

Meo M and Zumpano G (2005) On the optimal sensor placement techniques for a bridge structure. Engineering $\begin{array}{llll}\text { Structures, } & \text { Elsevier } & \text { 27(10): } & \text { 1488-1497. }\end{array}$ https://doi.org/10.1016/j.engstruct.2005.03.015

Milanese et al. (2008) Modeling and Detection of Joint Loosening using Output-Only Broad-Band Vibration Data. Structural Health Monitoring, SAGE 7(4): 309-328.

Mourad et al. (2012) Robust bounded area tracking in wireless sensor networks. Proceedings of $16^{\text {th }}$ IFAC symposium on system identification, Brussels, pp. 1097-1102. 
Papadimitriou C (2005) Pareto optimal sensor locations for structural identification. Computer Methods in Applied Mechanics and Engineering, Elsevier 194(12-16): 1655-1673. https://doi.org/10.1016/j.cma.2004.06.043

Yi TH and Li HN (2012) Methodology Developments in Sensor Placement for Health Monitoring of Civil Infrastructures. International Journal of Distributed Sensor Networks, Hindawi Publishing Corporation, doi:10.1155/2012/612726

Yuen KV and Kuok SC (2014) Efficient Bayesian sensor placement algorithm for structural identification: a general approach for multi - type sensory systems. Earthquake Engineering \& Structural Dynamics, Wiley 44: 757-774. https://doi.org/10.1002/eqe.2486

Zhou et al. (2013) Sensor placement for structural damage detection considering measurement uncertainties. Advances in Structural Engineering, SAGE 16(5): 899-907. https://doi.org/10.1260/1369-4332.16.5.899

Zuev KM and Katafygiotis LS (2011) Modified MetropolisHastings algorithm with delayed rejection. Probabilistic Engineering Mechanics, Elsevier 26(3): 405-412. https://doi.org/10.1016/j.probengmech.2010 